\title{
Estrategias comunicativas en la relación de padres e hijos adolescentes, 2018
}

\section{Communicative strategies in the relationship of parents and adolescent children, 2018}

\author{
Olga Natividad Arellanos Tafur \\ Rosario del Carmen Arellanos Tafur \\ Universidad César Vallejo, Perú
}

Autor para correspondencia: olgarellanost@gmail.com; charoare2@gmail.com

Fecha de recepción: 5 de febrero del 2019 - Fecha de aceptación: 15 de abril del 2019

Resumen: El siguiente artículo es de naturaleza teórica y tiene por objetivo presentar un análisis de la situación en la que se encuentran los padres de familia en relación con sus hijos adolescentes; así como, una herramienta para mejorar dicha relación familiar. La creación de este tipo de proyectos es importante, ya que presentan experiencias exitosas de otros países; los cuales, presentan programas que pueden ser aplicadas para evitar que se deteriore la comunicación entre padres e hijos adolescentes; ya que se encuentran en una etapa muy difícil. La metodología que se utilizó es de origen mixto y se recurrió a tres de sus técnicas que son la observación, la revisión bibliográfica y de campo. En la ejecución de los talleres se realizaron actividades formativas de interés tanto para padres e hijos que permitieron desarrollar estrategias para lograr una comunicación asertiva y de esta manera contribuir a la formación integral de los adolescentes y lograr un proyecto de vida.

Palabras claves: estrategias comunicativas y relación de padres a hijos

Abstract: The following article is of a theoretical nature and aims to present an analysis of the situation inwhich parents find themselves in relation to their adolescent children; as well as, a tool to improve this family relationship. The creation of this type of projects are important, since they present successful experiences from other countries; which, they present programs that can beapplied to avoid that the communication between parents and adolescent children is deteriorated; since they are in a very difficult stage. The methodology used was of mixed origin and three of its techniques were used: observation, bibliographic and field review. In the execution of the workshops formative activities of interest for both parents and children were carried out, which allowed developing strategies to achieve an assertive communication and in this way contribute to the integral formation of the adolescents and achieve a life project.

Keywords: communication strategies and parent-child relationship 


\section{Introducción}

La etapa de la adolescencia es el ciclo vital humano más difícil y problemático; debido a los cambios que sufren y muchas veces considerado menos comprendidas. El propósito de esta investigación es conocer estos cambios y ayudar a los padres de familia a tener una mejor comunicación con sus hijos.

La adolescencia es una etapa de transición que no tiene límites temporales fijos; pero, son significativos y abarca desde cambios biológicos hasta cambios de conducta y status social, dificultando de esta manera precisar sus límites de manera exacta. Bassat (2014).

En esta etapa surge una crisis; en ella, se produce una agudización en las contradicciones sobre las relaciones internas. Este período de transición; abarca entre los 10 y 19 años de edad. Comprende la pubertad (etapa de juventud hasta los 20 años), y la juventud (rasgos biológicos de la adolescencia en los adultos).

La adolescencia es una etapa del desarrollo del ser humano, en ella se evidencian cambios bruscos, psicológicos, biológicos y sociales; mayormente son rápidos, repentinos o demorados. Cuando es acelerado produce ansiedad y con ritmo demorado, zozobra e inquietud.

Las conductas de los padres de familia, generalmente no es el adecuado; no tienen una comunicación fluida, sus expresiones lastiman a los hijos en vez de corregirlos; esto hace que los hijos confíen más en sus amigos y no en sus padres.

En este artículo se detalla en primer lugar la importancia de la familia en la etapa de la adolescencia. En la segunda parte se da a conocer el manejo de conflictos en esta etapa. En la tercera etapa se analizará la relación entre padres e hijos adolescentes y la importancia de los talleres protectores.

Este tipo de análisis fue realizado en una Institución Educativa Nacional en el nivel secundaria; la cual, cuenta con adolescentes con problemas de conducta, además de ser considerada un asentamiento humano y en su mayoría pertenecen a familias disfuncionales y no tienen una buena relación con sus padres.

\section{La familia y la comunicación}

Rubio (2007) sostiene que "comunicar es percepción, crea expectativas y plantea exigencias". (p. 66).

La comunicación tiene como objetivo cambiar el comportamiento, actitudes o a hacer algo que no haría intencionalmente la persona a la cual se comunica algo.

Gallego (2006, p. 7) manifiesta que los símbolos verbales y no verbales y sus significados representan formas, patrones y sistemas comunicativos específicos expresándose cotidianamente de diferentes formas en la medida que las personas les dan sentido y significado a sus relaciones familiares. 
Cada familia establece sus propios códigos de comunicación, lo cual se manifiesta a través de sus relaciones. La comunicación constituye un elemento importante que determina las clases de relación que hace que sea único y singular en cada familia.

Mediante la comunicación se determinan roles, reglas actividades y tareas, desarrollando patrones comportamentales, por eso es necesario que los Padres de Familia comprendan los procesos comunicativos, ya que muchas investigaciones han demostrado que las familias que mantienen procesos comunicativos y relacionales dinámicos y proactivos, facilitan su actuar, el logro de sus metas y objetivos como colectivos. Así mejoran cada periodo sus metas para ir avanzando hacia una mejor calidad de vida.

Las familias donde la comunicación es fluida: Sus integrantes se involucran y se comprometen en los problemas; tomando decisiones en conjunto para buscar alternativas en solución. Cada integrante manifiesta una actitud de apertura y cálida tratando de entender el punto de vista de cada uno. También se promueve la expresión de emociones, sentimientos individuales, respetándose mutuamente, así cada integrante desarrolla capacidades para enfrentar situaciones y contradicciones que se le van presentando en la vida. Igualmente, cada integrante asume con responsabilidad las consecuencias de sus actos y comportamientos.

Asimismo, los integrantes desarrollan la capacidad de negociación. Frente a los problemas muestran flexibilidad y adaptabilidad.

\section{Problemas en la adolescencia}

Existe una información limitada sobre prácticas, comportamientos y conductas de riesgo en los adolescentes; lo cual, no permite tener una visión integral de la problemática. Esta comunidad está sometida a los riesgos por muchos factores; entre ellos, la ubicación geográfica, los tipos de familia, la presión de grupos, etc. ¿Influirá este riesgo específico en el comportamiento, prácticas y conductas de riesgo en los adolescentes?

Cabe resaltar que la mayoría de adolescentes consumen drogas, las cuales producen dependencia psicológica y física intensa. Al respecto, Moreno (2012), en su libro "Drogas: las 100 preguntas más frecuentes" manifiesta:

Los síntomas de la adicción varían en forma e intensidad de acuerdo con la droga empleada y el tiempo que lleva desarrollándose la dependencia, en todos los casos se debe a que se ha alterado el funcionamiento normal del sistema nerviosos" (p.51).

En otras palabras, las sustancias adictivas generan dependencia y permiten emociones como entusiasmo, alegría y serenidad. Son usados por los adolescentes para enfrentar momentos difíciles.

Para evitar que el problema se siga incrementando es importante considerar un modelo que se ajuste a las necesidades de los adolescentes y un programa de talleres protectores en la comunidad para mejorar la calidad de vida y la salud de los adolescentes. La prevención es primordial para evitar el daño y deterioro en jóvenes adolescentes y que estas conductas repercutan en la vida diaria y en el trabajo de nuestros jóvenes. Según las estadísticas mundiales 
los riesgos que se presentan están vinculados con el desarrollo científico y tecnológico, así como las condiciones socioeconómicas de cada nación. (Rosabal, 2015).

\section{Afectividad}

Domenech, E (2005, p. 185) sostiene que en la edad de la adolescencia los jóvenes inician nuevas e importantes relaciones afectivas con amigos y parejas, sin embargo, mantienen también vínculos afectivos con sus padres y éstos continúan siendo para ellos el principal apoyo emocional.

Este aspecto emocional es importante para que el adolescente pueda relacionarse con sus iguales estableciendo vínculos afectuosos de manera equilibrada y así poder desarrollarse.

Algunos padres de familia no toman en cuenta este aspecto y tratan a sus hijos adolescentes como jóvenes que no necesitan su apoyo emocional. Por el contrario, otros sobreprotegen a los jóvenes tratándolos como niños todavía. Ambos extremos afectan el desarrollo del adolescente pues por un lado se siente desprotegido y sólo, y por otro se siente sin libertad.

\section{Desarrollo de la Autonomía}

Como sostienen, Estevez, Jiménez y Musito (2011, p. 39) cualquier tensión que ocurra con alguno de sus integrantes de una familia repercutirá en toda la familia. En torno a esta situación es necesario establecer mecanismos como son las interacciones y reglas en la familia que facilite por un lado la unión de los miembros, a su vez permita el crecimiento personal de cada uno.

En cada etapa la familia debe organizarse y establecer normas de acuerdo a desarrollo de sus integrantes. Una etapa particular para el equilibrio de la familia es aquella en la cual uno de sus integrantes es adolescente.

En etapa debe renegociarse el grado de independencia y control de los hijos tomando en cuenta las nuevas demandas de los adolescentes. Aparece la necesidad de ampliar y ser flexibles con los límites familiares que permitan aceptar la independencia de los adolescentes dentro y fuera del núcleo familiar. En este sentido es importante que la familia integre los lazos afectivos, el sentido de pertenencia con la individualización de sus integrantes y su autonomía.

No debe confundirse la autonomía de los hijos con el rompimiento de las relaciones sino por el contrario es transformar las relaciones para lograr un equilibrio y reciprocidad. Esta autonomía está relacionada con acciones externas como permisos para salir fuera de casa, como las acciones internas como el tomar decisiones sobre su propia vida sin tener sentimientos de "desleales" a los padres.

Según Steinberg, como lo mencionan Estevez, Jiménez y Musito (2011, p. 41) la búsqueda de autonomía de un adolescente involucra tres tipos de autonomía: conductual, emocional y de valores. El proceso de para lograr la autonomía del adolescente de una manera 
positiva requiere de la actitud del propio adolescente y de la disposición de los padres. El adolescente debe adaptarse a los cambios bio psicosociales por los cuales está atravesando y configurar una imagen dejando de lado las definiciones que tenía de sí mismo cuando era niño.

Los padres deben tratar de conservar los vínculos establecidos con sus hijos, pues estos desean recibir su reconocimiento. Así pues, el adolescente desea que sus padres aprueben su identidad con los nuevos cambios que ha sufrido. Los Padres para ayudar a sus hijos adolescentes a ser autónomos tienen que dar la oportunidad a sus hijos a tomar decisiones que promueva el buen desarrollo de su identidad y su bienestar.

\section{Manejo de conflictos}

Cuando los adolescentes buscan tomar sus propias decisiones y tener autonomía, se originan los conflictos con sus padres.

Estévez, Jiménez y Musito (2011, p. 41) sostienen que los conflictos surgen cuando los adolescentes se sienten amenazados a perder su libertad y los padres al considerar que la libertada de los adolescentes amenaza la unión familiar. Muchos conflictos se generan por las percepciones polarizadas de padre e hijos.

Los hijos piensan que muchos aspectos de su vida, donde ellos pueden ahora como adolescentes pueden tomar decisiones, los padres no los dejan decidir. Ejemplo en la toma de decisiones de elección de su ropa. Muchos padres que decidían cuando eran niños que ropa es la adecuad, quieren continuar con esa actitud, generando un conflicto.

Pero los conflictos no deben considerarse como factores que promueven la desintegración familiar, por el contrario, debe ser el inicio para establecer el diálogo y llegar a un acuerdo. Lo importante de un conflicto es la forma como se ve y como se resuelve, en este sentido el afecto, la comunicación y el grado de disposición juegan un papel importante.

Así mismo el conflicto debe tomarse como una oportunidad para mejorar las relaciones, es así que tanto los adolescentes como los padres deben ser flexibles en sus posiciones, utilizar el diálogo para negociar.

Al negociar se debe tener una actitud de apertura a escuchar y a manifestar nuestra posición, mostrar flexibilidad a las diferentes posturas, ceder en los aspectos que se considere conveniente y luego establecer a la solución por consenso.

\section{Relación entre padres e hijos adolescentes}

Domenech, E. (2005, p. 186) Manifiesta que "Son varios los procesos y variables implicados en las relaciones de Padres e hijos".

Es importante destacar que en la etapa de la adolescencia los padres necesitan un asesoramiento relacionado a la tarea de crianza y educación de los hijos, ya que esta etapa puede resultar muy complicada para muchos padres y madres que se sentirán desorientados y confusos. 
Resulta necesario que dispongan de recursos que les apoyen en su tarea educativa, les transmitan conocimientos y estrategias para aumentar su competencia y mejorar su estilo parental y les sirvan para fortalecer sus nexos con la comunidad (Máiquez, Rodríguez y Rodrigo, 2004).

La estabilidad familiar consiste en saber comunicar de manera positiva, así puede existir mayor comprensión ante las adversidades. Jover y González (2013, p. 71) refiere "El amor se convierte en la base de la vida familiar". Si hay amor será posible crear vínculos seguros entre los integrantes garantizando un clima familiar saludable.

Los vínculos afectivos están presentes en todas las actividades de las personas con el entorno, facilitando la comunicación afectiva, el cuidado y buen trato en la relación de padres e hijos (Pérez y Arrázola, 2013).

\section{Comunicación}

Lanz, Lafrate, Rosanti y Scabini, como lo menciona Domenech, E. (2005, p. 190), manifiestan que la comunicación es importante en el periodo de transición que es la adolescencia, entre padres e hijos. Pues mediante la comunicación, padres y adolescentes llegan a negociar sus relaciones.

Muchos investigadores han establecido que en familias donde la comunicación es fluida y abierta, los conflictos son bajos y el control se da en un ambiente democrático. Los adolescentes fortalecen mejor su identidad teniendo la facultad de enfrentar a la realidad y los obstáculos que se les presenten. La comunicación familiar debe facilitar al adolescente el desarrollo de habilidades sociales que les permita afrontar situaciones problemáticas de forma constructiva y con efectividad.

Por otro lado, si la comunicación entre padres y adolescentes es buena, existirá mayor afecto, más flexibilidad para resolver los problemas y conflictos. El adolescente tendrá sentimientos positivos y de bienestar

\section{Desarrollo de la Autonomía}

Como sostienen Estevez, Jiménez y Musito (2011, p. 39) cualquier tensión que ocurra con alguno de sus integrantes de una familia repercutirá en toda la familia. En torno a esta situación es necesario establecer mecanismos como son las interacciones y reglas en la familia que facilite por un lado la unión de los miembros, a su vez permita el crecimiento personal de cada uno.

En cada etapa la familia debe organizarse y establecer normas de acuerdo a desarrollo de sus integrantes. Una etapa particular para el equilibrio de la familia es aquella en la cual uno de sus integrantes es adolescente.

En etapa debe renegociarse el grado de independencia y control de los hijos tomando en cuenta las nuevas demandas de los adolescentes. Aparece la necesidad de ampliar y ser flexibles con los límites familiares que permitan aceptar la independencia de los adolescentes dentro y 
fuera del núcleo familiar. En este sentido es importante que la familia integre los lazos afectivos, el sentido de pertenencia con la individualización de sus integrantes y su autonomía.

No debe confundirse la autonomía de los hijos con el rompimiento de las relaciones sino por el contrario es transformar las relaciones para lograr un equilibrio y reciprocidad. Esta autonomía está relacionada con acciones externas como permisos para salir fuera de casa, como las acciones internas como el tomar decisiones sobre su propia vida sin tener sentimientos de "desleales" a los padres.

Según Steinberg, como lo mencionan Estevez, Jiménez y Musito (2011, p. 41) la búsqueda de autonomía de un adolescente involucra tres tipos de autonomía: conductual, emocional y de valores. El proceso de para lograr la autonomía del adolescente de una manera positiva requiere de la actitud del propio adolescente y de la disposición de los padres. El adolescente debe adaptarse a los cambios biopsico sociales por los cuales está atravesando y configurar una imagen dejando de lado las definiciones que tenía de sí mismo cuando era niño. Los padres deben tratar de conservar los vínculos establecidos con sus hijos, pues estos desean recibir su reconocimiento. Así pues, el adolescente desea que sus padres aprueben su identidad con los nuevos cambios que ha sufrido.

El adolescente, para desarrollar su autonomía con un sentido positivo, debe armonizar aspectos personales y sociales, integrar la evolución individual y relacional.

En la familia se inicia la primera socialización, demostrándose las relaciones familiares decisivas para el desarrollo de la autonomía. La familia cumple un rol muy importante en la sociedad. La sociología entiende la familia como el primer núcleo de convivencia. La Pedagogía destaca su función educativa, cauce de integración biológica y social. Desde el punto de vista psicológico, es en la familia donde surgen los sentimientos, actitudes, motivación y conductas. La Psicología social la considera como eje sobre el que gira el ciclo vital, asegurando la continuidad de la sociedad y constituyendo el referente más importante para sus miembros (Palacios y Rodrigo, 2003).

Los Padres para ayudar a sus hijos adolescentes a ser autónomos tienen que dar la oportunidad a sus hijos a tomar decisiones que promueva el buen desarrollo de su identidad y su bienestar.

\section{Manejo de conflictos}

Cuando los adolescentes buscan tomar sus propias decisiones y tener autonomía, se originan los conflictos con sus padres.

Estévez, Jiménez y Musito (2011, p. 41) sostienen que los conflictos surgen cuando los adolescentes se sienten amenazados a perder su libertad y los padres al considerar que la libertada de los adolescentes amenaza la unión familiar. Muchos conflictos se generan por las percepciones polarizadas de padre e hijos. 
Los hijos piensan que muchos aspectos de su vida, donde ellos pueden ahora como adolescentes pueden tomar decisiones, los padres no los dejan decidir. Ejemplo en la toma de decisiones de elección de su ropa. Muchos padres que decidían cuando eran niños que ropa es la adecuad, quieren continuar con esa actitud, generando un conflicto.

Pero los conflictos no deben considerarse como factores que promueven la desintegración familiar, por el contrario, debe ser el inicio para establecer el diálogo y llegar a un acuerdo. Lo importante de un conflicto es la forma como se ve y como se resuelve, en este sentido el afecto, la comunicación y el grado de disposición juegan un papel importante.

Así mismo el conflicto debe tomarse como una oportunidad para mejorar las relaciones, es así que tanto los adolescentes como los padres deben ser flexibles en sus posiciones, utilizar el diálogo para negociar.

Al negociar se debe tener una actitud de apertura a escuchar y a manifestar nuestra posición, mostrar flexibilidad a las diferentes posturas, ceder en los aspectos que se considere conveniente y luego establecer a la solución por consenso.

En conclusión, los talleres de habilidades comunicativas ayudaron a mejorar las relaciones entre padres e hijos adolescentes.

\section{Método}

\section{Tipo de estudio y diseño de investigación}

En el presente proyecto se utilizará el diseño cuasi experimental que consiste en dos ediciones de la variable dependiente (Relación de Padres e hijos adolescentes) en dos momentos diferentes, un momento antes y un momento después, de la aplicación de los Talleres de estrategias comunicativas. Al respecto, Hernández, Fernández y Bautista (2014) sobre el diseño cuasi experimental dice que "A un grupo se le aplica una prueba previa al estímulo o tratamiento experimental después se le administra el tratamiento y finalmente se le aplica una prueba posterior al tratamiento" (p. 220). Es decir, como parte del estímulo se aplicará los Talleres estrategias comunicativas para lograr un efecto en la relación de padres e hijos adolescentes.

\section{Población, muestra y muestreo}

La población estará conformada por los 250 Padres de Familia de los estudiantes adolescentes de 4to y 5 to de secundaria de la Institución Educativa del distrito de Ate Vitarte. La muestra estadística estará conformada por 100 Padres de Familia, elegidos aleatoriamente.

\section{Técnicas e instrumentos de recolección de datos}

Técnica: Encuesta

Instrumento: Cuestionario

\section{Validación y confiabilidad de los instrumentos}

El instrumento será validado por juicio de expertos. 


\section{Procedimiento de recolección de datos}

Los datos serán recolectados aplicando el instrumento que mide la relación entre Padres e hijos adolescentes, se aplicará antes del inicio y al finalizar los talleres de estrategias comunicativas.

\section{Métodos de análisis de datos}

El análisis de datos cuantitativos se realizará tomando en cuenta los niveles de medición de la variable dependiente y mediante la estadística; que permitirán describir y poner de manifiesto las principales características de la variable, tomadas en dos momentos.

Para analizar la variable dependiente se utilizará del programa SPSS:

Estadística descriptiva: porcentajes en tablas y gráficas para presentar la distribución de los datos y tablas de contingencias.

Estadística inferencial: sirve para estimar parámetros y probar hipótesis, y se basa en la distribución muestral.

\section{Método}

El nivel de investigación es aplicativo; pues se aplicará una mejora con los talleres educativos. Diseño cuasi experimental, pues tiene dos mediciones antes y después. El tipo de estudio es prospectivo, longitudinal, comparativo, experimental. La población cuenta con 250 padres de familia en la Institución Educativa en el 2018 y se trabajó con una muestra de 120 padres de familia

Al respecto, Hernández, Fernández y Baptista (2014) sobre el diseño cuasi experimental dice que "A un grupo se le aplica una prueba previa al estímulo o tratamiento experimental, después se le administra el tratamiento y finalmente se le aplica una prueba posterior al tratamiento"

(p. 220).

Tabla: Diseño de pre-test y post-test.

con P. P. L.

$\mathbf{P}(\underline{R 1})=M_{1} P(R 1)=M_{1}$ Ge $=$ Grupo Experimental $=60$ padres Grupo $A$ $\mathrm{Ge}$ $\mathrm{F}$
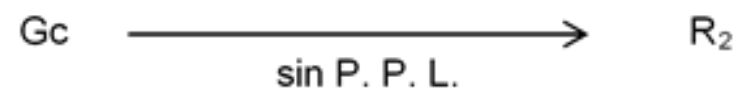

Gc $=$ Grupo Control $=60$ padres Grupo B$$
H_{1}: M_{1}>M_{2}
$$

$H_{\circ}: M_{1} \leq M_{2}$

$\mathrm{H}_{1}: \mathrm{M}_{1}>\mathrm{M}_{2}$

El enfoque empleado fue el cuantitativo porque se buscó medir las variables, particularmente la variable dependiente a fin de conocer su comportamiento en los sujetos en estudio. Este enfoque, según Valderrama (2015) asevera que "Usa la recolección y el análisis de los datos para contestar a la formulación del problema de investigación; utiliza, además, los métodos o técnicas estadísticas para contrastar la verdad o falsedad de la hipótesis” (p. 106). De 
esta manera, se contrastará la hipótesis mediante aplicación de técnicas estadísticas apropiadas al diseño cuasi experimental.

\section{Población}

Para el desarrollo de la investigación, se consideró como población de estudio a aquella conformada por los padres de familia de la Institución Educativa, en el año lectivo 2018, haciendo un total de 120, tal como se aprecia en el siguiente cuadro.

Tabla 2. Distribución de los padres de familia, Ate.

\begin{tabular}{lllll}
\hline \multirow{2}{*}{ Grado de estudios } & Sección & Sexo & & Total \\
& & M & F & \\
\hline \multirow{2}{*}{ Primer año } & A & 14 & 16 & 30 \\
\cline { 2 - 5 } & B & 11 & 13 & 24 \\
\hline Segundo año & A & 14 & 20 & 34 \\
\hline Tercer año & A & 16 & 16 & 32 \\
\hline Totales & & 55 & 65 & 120 \\
\hline
\end{tabular}

Fuente: Nominas de matrícula.

\section{Muestra}

Para obtener la muestra se utilizó el tipo de muestreo no probabilístico, permitiendo al investigador determinar la muestra según las condiciones establecidas por la organización educativa. De esta manera, la muestra se constituyó de 54 padres de familia del primer año, 34 en segundo y 32 en tercero de educación secundaria de la I.E., 60 al grupo control y 60 al grupo experimental. Los padres fueron elegidos aleatoriamente para la conformación de los grupos de estudio, considerándose el número de estudiantes, siendo divididos en dos grupos, un grupo experimental y un grupo de control.

\section{Resultados}

\section{Hipótesis general}

La aplicación de los talleres de estrategias comunicativas influye significativamente la relación de padres e hijos adolescentes en la Institución Educativa del distrito de Ate- Vitarte 2018.

\section{Hipótesis específicas}

La aplicación de los talleres de estrategias comunicativas influye significativamente en la comunicación afectiva entre padres e hijos adolescentes en la Institución Educativa del distrito de Ate- Vitarte 2018. 
La aplicación de los talleres de estrategias comunicativas influye significativamente en el desarrollo de la autonomía de los hijos adolescentes de la Institución Educativa del distrito de Ate- Vitarte 2018.

La aplicación de los talleres de estrategias comunicativas influye significativamente en el buen manejo de conflictos en la relación de padres e hijos adolescentes de la Institución Educativa del distrito de Ate- Vitarte 2018

Tabla 3. Estadísticos para una muestra.

\begin{tabular}{ccccc}
\hline & $\mathrm{N}$ & Media & $\begin{array}{c}\text { Desviación típica } \\
\text { Error tip de la } \\
\text { media }\end{array}$ \\
\hline Después / antes & 120 & 9,92 & 5,18 & 0,6 \\
\hline
\end{tabular}

Tabla 4. Prueba para una muestra.

\begin{tabular}{ccccccc}
\hline & $\mathrm{t}$ & Gl & Sig & $\begin{array}{c}\text { Diferencias de } \\
\text { medias }\end{array}$ & $\begin{array}{c}95 \% \text { intervalo de } \\
\text { confianza para la } \\
\text { diferencia } \\
\text { Inferior }\end{array}$ \\
\hline Después/ antes & 1,95 & 120 & 0,05 & 9,92 & $-0,07$ & 3,24 \\
\hline
\end{tabular}

Con una significancia de 0,05 , se valida la hipótesis con una diferencia de medias entre después y antes de 9,92.

Finalmente, es posible considerar que el estudio realizado presenta alcances que serán de contribución a las investigaciones a realizarse en el futuro sobre la aplicación de talleres de estrategias comunicativas en relación de padres e hijos adolescentes.

\section{Conclusiones}

Se logró determinar que el taller de estrategias comunicativas logró mejorar la relación de padres e hijos adolescentes en la Institución Educativa del distrito de Ate- Vitarte 2018. Se logró determinar que el taller de estrategias comunicativas logró una comunicación afectiva entre padres e hijos adolescentes en la Institución Educativa del distrito de Ate- Vitarte 2018.

Se logró determinar que el taller de estrategias comunicativas logró el desarrollo de la autonomía de los hijos adolescentes de la Institución Educativa del distrito de Ate- Vitarte 2018. Se logró determinar que los talleres de estrategias comunicativas lograron el buen manejo de conflictos en la relación de padres e hijos adolescentes de la Institución Educativa del distrito de Ate- Vitarte 2018

\section{Bibliografía}


Domenech, E. (2005). Actualizaciones en psicología y psicopatología de la adolescencia. Barcelona: Universidad Autónoma.

Estévez, E. Jiménez, T. y Musitu, G. (2007). Relaciones entre Padres e hijos adolescentes. Valencia: NAU libres.

Gallego, S. (2006). La comunicación y la familia. Colombia: Universidad de Caldas

García-Bacete, F. (2003). Las relaciones escuela-familia: un reto educativo. Infancia y Aprendizaje. Journal for the Study of Education and Development. España: Fundación Infancia y Adolescencia, vol. 26, no. 4, noviembre, pp. 425-437. [En línea] Disponible, consultada: septiembre de 2012.

Grupo Aprendizaje Emocional. (2007). Programa de educación emocional y prevención de la violencia. Primer ciclo de ESO. Alicante: Conselleria d’Educació. Recuperado de: https://convivencia.files.wordpress.com/2010/10/programa_ed-emocionalprevencion_violencia-1cesocaruana2004322p.pdf

Gutiérrez, M., Santos, A. M., Sancho, P., \& Galiana, L. (2013). Relaciones familiares, prácticas educativas de los padres y valores de los adolescentes angoleños. Psychology, Society \& Education, 5(1), 59-75. Recuperado de https://dialnet.unirioja.es/descarga/articulo/4489358.pdf

Hernández, G. (2004). Contextos educativos y acción tutorial. España:

Hernández, R. Fernández, C. y Baptista, P. (2014). Metodología de la investigación. México: Mac Graw Hill.

Jover, G., \& Gonzáles Martín, M. R. (2013). La revolución del amor como núcleo ético de la relación familiar. Revista Semestral del Departamento de Educación. Facultad de Filosofía y Letras, 25, 69-84.

Máiquez, L., Rodríguez, G. \& Rodrigo, J. (2004). Intervención psicopedagógica en el ámbito familiar: los programas de educación para padres. Infancia y Aprendizaje, 27, 403-406.

Medina, P. Alvarez, A. Hernández V. (2017). Comunicación entre padres e hijos adolescentes. Jóvenes en la ciencia -Revista de divulgación científica, 3 (2), México.

Mitjans L. (2005). Sexualidad y adolescencia. Revista Pediatría de Atención Primaria 7, Suplemento. 1, 89-95.

Moreno. K. (2012). Drogas: las 100 preguntas más frecuentes. Editorial: Trillas

Oliva, A. (2015). Desarrollo positivo adolescente. Madrid, España: Síntesis.

Palacios, J. y Rodrigo, M.J. (2003). Familia y desarrollo Humano. Editorial: Alianza 
Pérez, B., \& Arrázola, T. (2013). Vínculo Afectivo en la relación parentofilial como factor de calidad de vida. Revista Tendencia \& Retos, 18(1), 17-32.

Pérez O. Algunas reflexiones en torno a la comunicación en la formación. Revista electrónica pedagogía profesional; 2008.

Pérez, P.M., y Canovas, P. (2002). Valores y pautas de interacción familiar en la adolescencia (13-18 años). Maddd: Fundación Santa María

Rosabal, E., Romero N., Gaquín K. y Hernández R. (2015). Conductas de riesgo en los adolescentes. Ciencias médicas de las fuerzas revolucionarias. (La Habana). Revista cubana de Medicina Militar, 44(2):218-229

Rubio, M. (2007). La familia. España: Amat

Schmidt, V. Maglio, A. Messoulam, N. Molina, M. González, A. (2010). La Comunicación del Adolescente con Sus Padres: Construcción y Validación de Una Escala desde un Enfoque Mixto. Revista Interamericana de Sicología, 44 (2).

Sobrino, L. (2008). Niveles de comunicación familiar entre padres e hijos. Av. Psicol (16)1, EnDic. Perú. 